$\underline{\text { Research article }}$

\title{
An Outbreak of Hepatitis B in a Rural Area of West Bengal, India
}

\author{
DK Biswas, ${ }^{1}$ R Bhunia, ${ }^{2}$ P Das ${ }^{3}$ \\ Sri Lankan Journal of Infectious Diseases 2015 Vol.5 (2):51-57 \\ DOI: http://dx.doi.org/10.4038/sljid.v5i2.8077
}

\begin{abstract}
Introduction

There were limited data on outbreaks of hepatitis in rural settings in India. We describe an outbreak of Hepatitis $B$ virus infection to confirm the existence of an outbreak of Hepatitis B in a rural area of West Bengal, India and to determine risk factors for the outbreak and recommend preventive measures.
\end{abstract}

\section{Methods}

We surveyed the entire village retrospectively followed by a cross sectional case control study. We selected Hepatitis B surface Antigen (HBsAg) positive patients as cases and recruited neighbours as controls with a ratio of 1:2. The study was undertaken between November 2013 and June 2014. We collected blood samples which were sent to the laboratory for virological examination. Data were analyzed with Epi-info and Excel software.

\section{Results}

We surveyed 2170 residents. Of the 1085 residents in the risk population, 54 were tested for hepatitis B and 13 identified as hepatitis B positive. Among them 77\% (10) were men. Overall attack rate was 0.59\% (13/1085). One patient died (Case Fatality Ratio $=7.7 \%$ ). Of the tested population, 53\% (7) were illiterate and 39\% (5) were migrant labour by occupation. Compared with controls, significantly higher number of cases were given injection by local medical practitioners (LMP) (OR 7.50 95\% CI 1.19-78.93) and had unsafe sex with sex workers (OR 15.63, 95\% CI 1.34-767.41).

\section{Conclusion}

Injections given by LMPs and unsafe sex with sex workers were risk factors for the outbreak. Awareness programmes were carried out for LMPs on safe injection practice and villagers on safe sex and blood borne diseases. Hepatitis B vaccination was recommended for infants and adults.

Key words : Hepatitis B, Unsafe injection, Rural, India

\section{Introduction:}

Hepatitis B infection, formerly known as "Serum Hepatitis" affects the liver and produces acute and chronic liver diseases. ${ }^{1}$ Globally, in 2012, it was estimated that 240 million people were chronically infected with Hepatitis B virus (HBV). ${ }^{2}$ More than 780,000 people die each year due to consequences of Hepatitis B diseases. ${ }^{3}$ High concentration of $\mathrm{HBV}$ is present in blood, serum and serum exudates and moderate concentration of HBV is present in saliva, vaginal fluid and seminal fluid. HBV spreads through the parental route and sexual intercourse. Risk of development of chronic liver disease depends on the age of acquiring

\footnotetext{
${ }^{1}$ Purba Medinipur, West Bengal, India

${ }^{2}$ Lady Dufferin Victoria Hospital, Kolkata, West Bengal, India

${ }^{3}$ Department of Community Medicine, College of Medicine \& Sagore Dutta Hospital, Kamarhati, Kolkata, West Bengal, India

Address for correspondence: Dr. Dilip Kumar Biswas Welcome Housing, 228 Ashokegarh, Kolkata-700108, Pin: 700108 West Bengal, India Tel: 919433200804 Email: dilipbiswas29@ gmail.com
} 
HBV infection. Children less than six years of age who become infected with HBV are more likely to develop chronic liver disease. The risk of chronic liver diseases in HBV infected infants infected is $80-90 \%$ and $30-$ $50 \%$ in children between one and six years. ${ }^{4}$ However, the risk of developing chronic liver disease is decreased to $2 \%$ in older children (> 6years) and adults. ${ }^{5}$

Transmission of HBV can be prevented by the administration of Hepatitis B vaccine to infants and adults. ${ }^{6}$ Hepatitis B outbreaks could result from lack of injection safety by health care providers and percutaneous and mucosal exposure to blood of the infected person. Finger prick injuries have been reported with increasing frequency. ${ }^{7,8,9}$ In the South East Asian Region (SEAR' 2004), 80 million HBV carriers were estimated with HBV prevalence $\geq 8 \%$. ${ }^{10}$ India has an intermediate endemicity of HBV with prevalence of $2-7 \%$ with mean surface antigen carrier rate of $4.7 \% .^{11,12}$

Health workers informed the district surveillance officer of the death of a patient and an increased number of patients with jaundice in the village of Bhogpurr Maktabpara, under Panskura-II block of Purba Medinipur district, West Bengal, India in November 2013. We investigated the outbreak with the following objectives

\section{Objectives}
i. Confirm the existence of an outbreak
ii. Determine risk factors
iii. Recommend preventive measures.

\section{Methods}

\section{Descriptive epidemiology:}

We searched for jaundice case-patients in the village. We reviewed the data of jaundice case-patients for the past three years at the Sub-center where the study village was situated. Sub-center (SC) is the grass route level of health service delivery center. In a plains area, a SC population will be at least 5000 and in hilly and riverine remote areas, the SC population will be 2000 . We observed the migration and population influx of the village. We checked for any change of case definition and surveillance system in the village. We investigated all the case-patients by house to house survey. We defined a suspected case as a resident of the village of Bhogpur who had jaundice, abdominal pain, nausea, vomiting, tiredness, loss of appetite and dark coloured urine and confirmed case as a suspected case who was positive for HBsAg. We interviewed all case-patients, their neighbours and Local Medical Practitioners (LMPs). We generated the hypothesis that unsafe injection practice by the LMPs was the risk factor for this Hepatitis B outbreak.

\section{Analytical study:}

A case control study was carried out at Bhogpur Maktabpara village of Purba Medinipur district, West Bengal; India between November 2013 and June 2014. We selected as cases those who were laboratory confirmed as HBsAg positive in the study period among the age group of $14-60$ years. Neighbours of the cases during the study period whose blood was negative for HBsAg were selected as controls with a ratio of 1:2 (one case: two controls).

\section{Data collection:}

We constructed a questionnaire for data collection. The questionnaire contained several sections such as sociodemographic status (information related to age, sex, marital status, education, family income, occupation, religion, history of migration for work), risk factors (information related to exposures such as injuries to the body, history of injection, intravenous blood or saline, contact with a jaundiced patient, tattooing over the body surface and circumcision. Questions were also asked related to sexual exposure, sexual partners, and use of drugs by injections. We trained six health workers on data collection. The health workers collected data by interviewing the cases and controls as well as local LMPs with the pretested questionnaires. 
Data analysis: Data were analyzed in Epi-info 3.5.1 version and excel soft ware for rate and ratio.

\section{Laboratory Methods:}

Blood specimens were collected and centrifuged for separation of serum and sent to the Virology Department, School of Tropical Medicine, Kolkata for virological tests maintaining the cold chain. Blood specimens were also sent for human immunodeficiency virus (HIV) testing to the Integrated Counselling and Testing Centre (ICTC) laboratory.

\section{Environmental investigations:}

We visited the village and observed treatment seeking behaviour, water supply system, sanitation system, social, cultural and behaviour practices. We interviewed LMPs and observed their treatment system. Most of the villagers seek minor medical help from the LMPs who are unqualified doctors. We also visited hair cutting salons located in this village and observed their practice.

The documented total population of the village was 2170 and the population aged between $16-60$ years was $1085 .^{13}$

\section{Results:}

\section{Descriptive Epidemiology:}

Table 1: Socio-demographic characteristics of outbreak of Hepatitis B at Bhogpur Maktabpara village of Purba Medinipur district, West Bengal; India

\begin{tabular}{llcc}
\hline Characteristics & & Number & \% \\
\hline Age group in & $1-15$ & 0 & 0 \\
years & $16-30$ & 8 & 61.5 \\
& $31-45$ & 4 & 30.8 \\
Education & $46-60$ & 1 & 7.7 \\
& Illiterate & 7 & 53.8 \\
& 1-5 Standard & 4 & 30.8 \\
Occupation & 6-10 Standard & 2 & 15.4 \\
& Cultivation & 1 & 7.7 \\
& Petty Business & 5 & 38.5 \\
& Student & 1 & 7.7 \\
Family Income & Home maker & 1 & 7.7 \\
(Rupees) & Labour (including & 5 & 38.5 \\
Religion & mp to Rs: $3000 /$ - per & 13 & 100 \\
Gender & month & & \\
& Muslim & 13 & 100 \\
& Male & 10 & 76.9 \\
& Female & 3 & 23.1 \\
\hline
\end{tabular}

We surveyed the total population $(n=2170)$ of the village of Bhogpur Maktabpara, Purba Medinipur district between June 2013 and July 2014 and identified the population aged between 16-60 years $(n=1085)$ as the population at risk of developing hepatitis $\mathrm{B}$ infection. Of this risk population, $\mathrm{HBsAg}$ was tested in 54 cases of whom 13 were identified as being HBsAg positive.

The overall attack rate was $1.19 \%$ (13/1085). Among them, 10 (77\%) were men. Seven (54\%) of the 13 patients were illiterate and $5(39 \%)$ were migrant labour. Their monthly income was less than Rs: 3000/(Three Thousand Indian Rupee) and all of them were Muslim by religion. (Table 1)

We reviewed the Hepatitis $B$ case records of the village in the last three years. There were no reported Hepatitis B cases during this period. There was no recent population influx in this village. 
Villagers preferred treatment of minor ailments such as injuries and fever from the LMPs. The observation was made that LMPs treated patients with injectable medicine to ensure quick recovery. Of the three LMP, two used glass syringes and reused plastic syringes. There was no arrangement of sterilization sets. It was also observed that needles and syringes were shared when giving injections to patients.

\section{Clinical Presentation:}

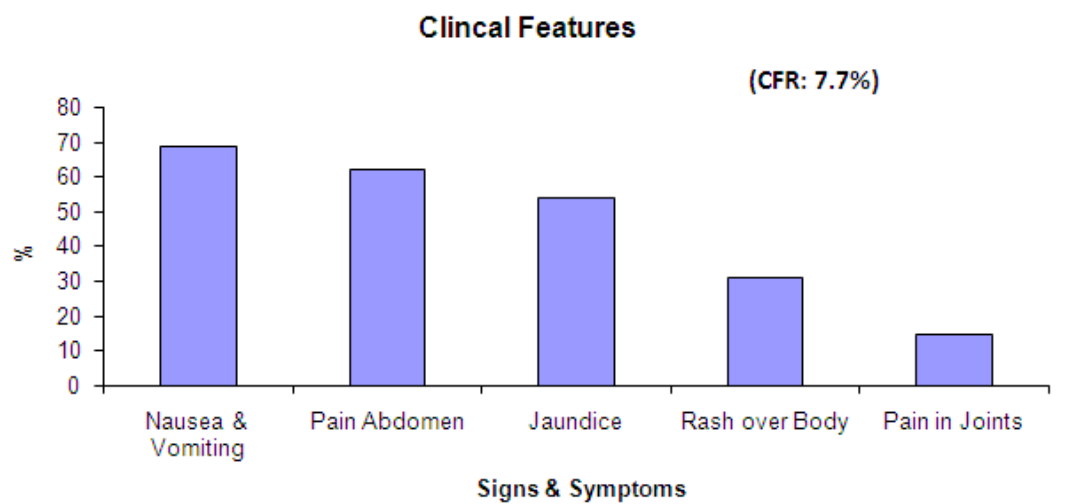

Figure 1

Clinical features of Hepatitis B outbreak at Bhogpur Maktabpara village of Purba Medinipur district, West Bengal; India 2014
Case-patients had symptoms of nausea and vomiting (69\%), abdominal pain $(62 \%)$, jaundice (54\%), rash over the body (31\%) and joints pain $(15 \%)$. One patient died due to hepatitis $\mathrm{B}$ related disease (case fatality ratio: 7.7\%). (Figure 1)

All cases were treated locally by LMPs first. One patient admitted first at Nursing home at Tamluk, Purba Medinipur district and thereafter transferred at IPGMR \& SSKM hospital, Kolkata where he expired. None of the patients had received Hepatitis B vaccination.

\section{Laboratory Findings:}

All the 13 case-patients were positive for HBsAg. Three case-patients had high serum bilirubin and serum glutamic pyruvic transaminase (SGPT) level. None of the case patients were found reactive for HIV. (Table 2)

Table 2: Laboratory test status of Hepatitis B outbreak at Bhogpur Maktabpara village of Purba Medinipur district, West Bengal; India 2014

\begin{tabular}{llllll}
\hline Age & Gender & Date of onset & $\begin{array}{c}\text { HBsAg B } \\
\text { result }\end{array}$ & $\begin{array}{l}\text { HIV AB } \\
\text { result }\end{array}$ & Out come \\
\hline 18 & Male & 02.11 .13 & Positive & Negative & Expired \\
45 & Female & 21.12 .13 & Positive & Negative & Improved \\
28 & Male & 22.12 .13 & Positive & Negative & Improved \\
35 & Male & 29.11 .13 & Positive & Negative & Improved \\
23 & Male & 29.12 .13 & Positive & Negative & Improved \\
27 & Male & 05.01 .14 & Positive & Negative & Improved \\
32 & Male & 07.01 .14 & Positive & Negative & Improved \\
28 & Male & 11.01 .14 & Positive & Negative & Improved \\
59 & Male & 17.01 .14 & Positive & Negative & Improved \\
50 & Male & 11.01 .14 & Positive & Negative & Improved \\
41 & Male & 12.01 .14 & Positive & Negative & Improved \\
16 & Female & 21.01 .14 & Positive & Negative & Improved \\
58 & Male & 23.02 .14 & Positive & Negative & Improved \\
\hline
\end{tabular}




\section{Case Control Study:}

All the confirmed 13 case-patients and 26 controls were recruited for the study with the ratio of 1:2. Median age of case-patients was 28 years with a range $16-59$ years and controls were 29 years ranging from 15 to 60 years. $77 \%(10 / 13)$ of the cases were men and 50\% (13/26) of the controls were women. Compared to controls, cases had significantly more exposure to shared needles and syringes by LMPs (OR 7.50, 95\% CI 1.19 - 78.93) and unsafe sex with sex workers (OR 15.63, 95\% CI 1.34 - 767.41) (Table 3)

Table 3: Risk factors of Hepatitis B outbreak at Bhogpur Maktabpara village of Purba Medinipur district, West Bengal; India 2014

\begin{tabular}{lcccccc}
\hline Risk factors & Case & \% & Control & \% & OR & 95\% CI \\
\hline Had injuries during previous year & 12 & 92 & 22 & 85 & 2.18 & $0.18-116.27$ \\
$\begin{array}{l}\text { Shared needles and syringes administered } \\
\text { by LMPs }\end{array}$ & 11 & 85 & 11 & 55 & 7.50 & $1.19-78.93$ \\
Unsafe sex with sex workers & 5 & 39 & 1 & 4 & 15.63 & $1.34-767.41$ \\
Contacted with jaundice cases & 9 & 69 & 5 & 19 & 9.45 & $2.04-43.60$ \\
\hline
\end{tabular}

\section{Environmental Investigations:}

Most residents of the village were Muslim by religion (98\%). Their occupation was mostly cultivation. The villagers sought medical treatment from the LMPs. Eleven of 13 case patients had shared needle, syringes during treatment. The LMPs used injectable medicines for quick response and reused needles and syringes without sterilization. They had no adequate knowledge about injection safety. 38\% (5/13) shared experiences of unsafe sex with commercial sex workers.

\section{Preventive measures}

We recommended Hepatitis B vaccination for adults and strengthening of the routine surveillance system. We arranged awareness generation programmes on hepatitis involving LMPs and villagers with special emphasis on use of disposable syringes, hepatitis B virus, hepatitis B vaccination and safe sex practices. We communicated the findings of our investigation to the district health officials and presented the findings in the State Integrated Diseases Surveillance Project (IDSP) workshops.

\section{Discussion:}

Epidemiological findings and laboratory results confirmed an outbreak of Hepatitis B. More men were affected than women. Unsafe sex with sex workers and unsafe injection practice (reuse of unsterile needles and syringes) by LMPs was the most likely ways in which Hepatitis B virus transmission occurred in this outbreak..

In rural areas, villagers usually sought treatment from the LMPs who were observed to reuse needles and syringes while administering injections. None of the tested group were positive for HIV even though they had unsafe sex with sex workers. A similar outbreak occurred at Modasa Town, India. ${ }^{14}$ Unsafe sex practices with sex workers (if infectious) might be risk of transmission of Hepatitis B as shown in this study. ${ }^{15}$

Hepatitis B vaccine administration was not included in the Universal Immunization Programme (UIP) in West Bengal until 2007-2008. Since then infants have been vaccinated with full doses. ${ }^{16}$ Adults have not been immunized as it was not included in UIP previously and vaccine is costly. This was the reasons of Hepatitis B non-vaccination among the villagers.

A previous study in Gujrat described an outbreak linked to use of unsafe injection practices by local unqualified doctors with a case fatality ratio of $46.7 \%{ }^{17}$ whereas the case fatality ratio in the current study was less than 7.7\%. Another study in the Sabarbanth district of Gujrat showed a breach of injection safety procedures 
practiced by unqualified doctors (in the form of reuse of injection syringe and needle without proper sterilization, multiple injections from the same vial with same needle/ syringe) which led to a Hepatitis B outbreak. ${ }^{18}$ Similar unsatisfactory practices by LMPs (unqualified doctor) was also observed in the current study.

Several studies have shown the risk of transmission of Hepatitis B in the community due to needles, syringes injuries. ${ }^{19,20}$ Contaminated needle was one of the commonest modes of transmission of HBV. LMPs had no or inadequate knowledge of safe injection practices. These unsafe practices could be reduced by providing knowledge regarding sterilization of needles/ syringes and increase general knowledge about infectious disease transmission to LMPs.

\section{Conclusion:}

Possibly, unsafe injection practices followed by LMPs led the Hepatitis B outbreak. LMPs, particularly in rural areas need to be trained on safe injection practices. Use of auto-disposable syringes at government and private sector may help prevent transmission of viral diseases such as hepatitis B, C and HIV. It is also necessary to increase awareness of routes of transmission of Hepatitis B as well as improve surveillance and immunization of those at risk.

\section{References:}

1. World Health Organization. Hepatitis B. Fact Sheet $N^{0}$ 204.Updated June 2014. Available from: www.who.int/mediacenter/factsheets/fs204/en

2. World Health Organization. Guidance on prevention of viral Hepatitis B and C among people who inject drugs. 2012. Available from: www.who.int/hib/pub/guidence/hepatitis/en

3. World Health Organization. Hepatitis B. Fact Sheet $\mathrm{N}^{0}$ 204.Updated June 2014. Available from: www.who.int/mediacenter/factsheets/fs204/en

4. World Health Organization (WHO). Fact Sheet No 204, Updated July 2015. Available from: http://www.who.int/mediacentre/factsheets/fs204/en/

5. Hyams KC. Risks of chronicity following acute hepatitis B virus infection: a review. Clin Infect Dis 1995; 20: 992-1000 doi : http://dx.doi.org/10.1093/clinids/20.4.992

6. Hepatitis B Vaccine. Center for Disease Control. Vaccine Information Statement. Available from: http://www.cdc.gov/vaccines/hcp/vis/vis-statements/hep-b.pdf

7. Gupta E, Bajpai M, Sharma P, Shah A, Sarin SK. Unsafe injection practices: A potential weapon for the outbreak of blood borne viruses in the community. Ann Med Health Sci Res. 2013; 3(2):177-181. doi : http://dx.doi.org/10.4103/2141-9248.113657

8. Bender TJ, Wise ME, Utah O, Moorman AC, Sharapov U, et al. Outbreak of Hepatitis B virus infections associated with assisted monitoring of blood glucose in an assisted living facility-Virginia, 2010. PLoS ONE 7(12): e50012. doi:10.1371/journal.pone.0050012

9. Centers for Disease Control and Prevention (2012) Healthcare-Associated Hepatitis B and C. Outbreaks reported to the Centers for Disease Control and Prevention (CDC) in 2008-2011. Available from: http://www.cdc.gov/ injectionsafety/blood-glucose-monitoring.html.

10. World Health Organization. Hepatitis B vaccines. WHO position paper. Weekly Epidemiological Record 2004; 79(28):(253-64). Available from: http://www.who.int/immunization_delivery/adc/hepb_wer.pdf

11. Aggarwal R, Ranjan P. Preventing and treating hepatitis B infection. Br Med J 2004; 329:1080-1086. doi : http://dx.doi.org/10.1136/bmj.329.7474.1080

12. Lai CL, Ratziu V, Yuen MF, Poynard T. Viral hepatitis B. Lancet 2003; 362:2089-2094. doi : http://dx.doi.org/10.1016/S0140-6736(03)15108-2

13. Census of India 2011, Available from: www.cnsusindiagov.in/2011

14. Patel DA, Praveg Gupta A, Kinaviwala DM, et al.. An investigation of an outbreak of viral Hepatitis B in Modasa Town, Gujrat. India. J Glob infect Dis 2012; 4(1):55-59 doi : http://dx.doi.org/10.4103/0974-777X.93762

15. Hepatitis A, B, C. AVERT. Available from: www.avert.org/hepatits-b-c.hpt 
16. Operational Guideline for Hepatitis B Vaccine Introduction in the Universal Immunization Programme. Ministry of Health \& Family Welfare. Government of India. New Delhi 2011. Available from: http://www.searo.who.int/india/topics/routine_immunization/Operational_Guidelines_for_HepatitisB_vaccine_in troduction_in_UIP_2011.pdf?ua=1

17. Singh J, Bhatia R, Gandhi JC, et al. Outbreak of viral hepatitis B in a rural community in India linked to inadequately sterilized needles and syringes. Bull World Health Organ 1998; 76:93-98. No doi

18. Dash B, Das RR. Outbreak of hepatitis B in Sabarkantha district of Gujarat: A case-control study. Int J Med Public Health 2013; 3:119-121. doi : http://dx.doi.org/10.4103/2230-8598.115188

19. Res S, Bowden FJ. Acute hepatitis B infection following a community-acquired needle sticks injury. $J$ Infect 2011; 62(6):487-489. doi : http://dx.doi.org/10.1016/j.jinf.2011.04.002

20. Garcia-Algar O, Vall O. Hepatitis B virus infection from a needle stick. Pediatr infect Dis J 1997;16(11):10991103. doi : http://dx.doi.org/10.1097/00006454-199711000-00027 